\title{
LEGISLACIÓN BÁSICA DE PROTECCIÓN DEL MEDIO AMBIENTE (PRIMER SEMESTRE 2021)
}

\author{
LAURA Presicce \\ Investigadora Predoctoral \\ Departamento de Derecho Público - Universitat Rovira i Virgili \\ laura.presicce@urv.cat
}

SUMARIO: 1. Introducción. 2. Medidas para la reactivación económica y para hacer frente a la vulnerabilidad social. 3. Novedades normativas en ámbitos sectoriales. 3.1. En materia de energía eléctrica. 3.2. En materia de contaminación atmosférica. 3.3. En materia de residuos. 3.4. En materia de instalaciones térmicas en edificios. 4. Ulteriores disposiciones de interés para la protección del medio ambiente.

\section{INTRODUCCIÓN}

El 25 de octubre de 2020, a través del Real Decreto 926/2020', el Gobierno declaró un ulterior estado de alarma en todo el territorio nacional para contener la propagación del Coronavirus. El estado de alarma, posteriormente prorrogado hasta el 9 de mayo de $2021^{2}$, estuvo vigente, pues, por todo el periodo que cubre esta crónica (1 de octubre de 2020 a 31 de marzo de 2021).

En dicho plazo de tiempo, a nivel estatal, se han aprobado un importante numero de Real Decretos-leyes para la reactivación económica y para hacer frente a la vulnerabilidad social provocada por la pandemia. Algunos de ellos, que introducen medidas en el ámbito medioambiental y energético, serán objeto de

\footnotetext{
${ }^{1}$ Real Decreto 926/2020, de 25 de octubre, por el que se declara el estado de alarma para contener la propagación de infecciones causadas por el SARS- CoV-2. BOE núm. 282, de 25 de octubre de 2020.

2 Real Decreto 956/2020, de 3 de noviembre, por el que se prorroga el estado de alarma declarado por el Real Decreto 926/2020, de 25 de octubre, por el que se declara el estado de alarma para contener la propagación de infecciones causadas por el SARS-CoV-2. BOE núm. 291 de 4 de noviembre de 2020.
} 
análisis en los epígrafes siguientes. Entre todos, merece una especial atención el Real Decreto-ley 36/2020, de 30 de diciembre, por el que se aprueban medidas urgentes para la modernización de la Administración Pública y para la ejecución del Plan de Recuperación, Transformación y Resiliencia.

Por otro lado, en cuanto a la normativa sectorial aprobada en los meses que cubre la crónica, hay que mencionar, en el ámbito de la energía eléctrica, la aprobación del estatuto de los consumidores electrointensivos y la normativa que regula el acceso y conexión a las redes de transporte y distribución de energía eléctrica. Asimismo, de especial interés resulta, en materia de contaminación atmosférica, la normativa aprobada para intensificar la reducción de GEI en el marco del régimen del comercio de derechos de emisión de gases de efecto invernadero, relativa al nuevo periodo de comercio 2021-2030.

\section{MEDIDAS PARA LA REACTIVACIÓN ECONÓMICA Y PARA HACER FRENTE A LA VULNERABILIDAD SOCIAL}

Para hacer frente a la fuerte contracción económica provocada a raíz de las restricciones a la actividad y la movilidad, necesarias para controlar la transmisión del Covid-19, el Gobierno, en el periodo de referencia de esta crónica, ha continuado aprobando una serie disposiciones de apoyo al tejido productivo y social, así como normativas para hacer frente a situaciones de vulnerabilidad social.

Entre todas, destacamos las siguientes:

a) El Real Decreto-ley 34/2020, de 17 de noviembre, de medidas urgentes de apoyo a la solvencia empresarial y al sector energético, y en materia tributaria ${ }^{3}$.

A través del Capítulo II de este Real Decreto-ley se incorporan a nuestro ordenamiento jurídico los cambios legislativos introducidos por la Directiva (UE) 2019/692 del Parlamento Europeo y del Consejo, de 17 de abril de 2019, por la que se modifica la Directiva 2009/73/CE sobre normas comunes para el mercado

${ }^{3}$ BOE núm. 303, de 18 de noviembre de 2020. 
interior de gas natural, con el objeto de reducir los obstáculos a la plena realización del mercado interior de gas natural que se derivan de la inaplicabilidad de las normas del mercado de la Unión a los gasoductos de transporte con destino u origen en países no pertenecientes a la Unión Europea. En esta senda, la norma prevé una exención relativa a la separación de propiedad entre la actividad de transporte y comercialización o producción de gas natural y de acceso regulado y no discriminatorio a sus instalaciones. Asimismo, se pretende garantizar la posibilidad, para los titulares de gasoductos de interconexión con países no pertenecientes a la Unión Europea, y que ya se encuentren construidos a la fecha de entrada en vigor de la Directiva, de adoptar un modelo que contemple la figura del Gestor Independiente para la gestión de estos. Finalmente, se regula un procedimiento de negociación con terceros países, respecto de las redes de transporte de gas natural, bajo la supervisión de la Comisión Europea.

En cuanto al sector eléctrico, en el mismo capítulo, se establece un incremento del límite máximo de las transferencias al sistema eléctrico provenientes de los ingresos de las subastas de derechos de emisión de gases de efecto invernadero, con los límites del noventa por ciento de la recaudación total y hasta un máximo de mil millones de euros. También se incrementa el límite máximo de las transferencias a actuaciones de lucha contra el cambio climático provenientes de los ingresos de las subastas de derechos de emisión de gases de efecto invernadero, con los límites del diez por ciento de la recaudación total y hasta un máximo de cien millones de euros.

El Real Decreto-ley modifica, entre otras, la Ley 34/1998, de 7 de octubre, del sector de hidrocarburos.

b) El Real Decreto-ley 37/2020, de 22 de diciembre, de medidas urgentes para hacer frente a las situaciones de vulnerabilidad social y económica en el ámbito de la vivienda y en materia de transportes y el Real Decretoley 1/2021, de 19 de enero, de protección de los consumidores y usuarios frente a situaciones de vulnerabilidad social y económica ${ }^{4}$.

${ }^{4}$ BOE núm. 334, de 23 de diciembre de 2020. 
La DA 4a del mencionado Real Decreto-ley establece la prohibición, mientras dure el actual estado de alarma, de suspender el suministro de energía eléctrica, gas natural y agua a aquellos consumidores en los que concurra la condición de consumidor vulnerable, vulnerable severo o en riesgo de exclusión social definidas en los artículos 3 y 4 del Real Decreto 897/2017, de 6 de octubre, por el que se regula la figura del consumidor vulnerable, el bono social y otras medidas de protección para los consumidores domésticos. Para acreditar la condición de consumidor vulnerable ante las empresas suministradoras de gas natural y agua es suficiente presentar de la última factura de electricidad en la que se refleje la percepción del bono social de electricidad.

c) El Real Decreto-ley 36/2020, de 30 de diciembre, por el que se aprueban medidas urgentes para la modernización de la Administración Pública y para la ejecución del Plan de Recuperación, Transformación y Resiliencia 5 .

El Real Decreto-ley 36/2020 merece un apunte destacado. Para ubicarlo correctamente es necesario recordar que, el 21 de julio de 2020, el Consejo Europeo acordó un paquete de medidas de gran alcance entre las cuales el Instrumento Europeo de Recuperación («Next Generation EU»), que proporcionará a España unos 140.000 millones de euros en forma de transferencias y préstamos para el periodo 2021-2026. El objetivo de la UE, a través de dicha movilización de recursos, es el de impulsar una recuperación económica de los países miembros que, a la vez, sea sostenible y acelere la transición ecológica y digital.

La transición hacia una economía y sociedad climáticamente neutras, sostenibles, circulares, respetuosas con los límites impuestos por el medio natural, y eficientes en el uso de recursos, es, por ende, uno de los objetivos básicos de los fondos Next Generation UE.

\footnotetext{
${ }^{5}$ BOE núm. 341, de 31 de diciembre de 2020. [Resolución de 28 de enero de 2021, del Congreso de los Diputados, por la que se ordena la publicación del Acuerdo de convalidación del Real Decreto-ley 36/2020, de 30 de diciembre, por el que se aprueban medidas urgentes para la modernización de la Administración Pública y para la ejecución del Plan de Recuperación, Transformación y Resiliencia, BOE núm. 30, de 4 de febrero de 2021].
} 
El Gobierno presentó el 7 de octubre el Plan de Recuperación, Transformación y Resiliencia que permitirá a España, conforme a las directrices de la Unión Europea, acogerse a la financiación de los diferentes mecanismos del Instrumento Europeo de Recuperación.

En esta senda, la aprobación del Real Decreto-ley objeto de análisis se justifica por la necesidad de contar con una Administración pública moderna y ágil que sepa diseñar, ejecutar y fiscalizar los proyectos que constituyen el Plan de Recuperación Transformación y Resiliencia, para absorber con éxito los fondos europeos del Instrumento Europeo de Recuperación.

Las medidas más importantes que el Real Decreto-ley objeto de análisis establece en cuanto a la materia medioambiental se refieren a la evaluación ambiental de los proyectos del Plan Recuperación, Transformación y Resiliencia.

El Capítulo VI del Título IV y la DF 3aㅡ modifican la Ley 21/2013, de 9 de diciembre, de Evaluación ambiental (LEA) con el fin de dotar de mayor agilidad y seguridad jurídica a los procedimientos de evaluación ambiental, y facilitar la tramitación de proyectos que permitan impulsar la reactivación económica, especialmente en el marco de los planes de inversión en las áreas de las tecnologías limpias, el uso de energías renovables y de eficiencia energética del Mecanismo de Recuperación y Resiliencia de la Unión Europea, al tiempo que se garantiza la protección del medio ambiente.

Recordamos, a tal propósito, que ya a través del Real Decreto-ley 23/2020, de 23 de junio, por el que se aprueban medidas en materia de energía y en otros ámbitos para la reactivación económica, se había llevado a cabo una modificación de la LEA para dotar al procedimiento de evaluación ambiental de una mayor agilidad y seguridad jurídica, facilitando la tramitación de proyectos que permitan impulsar la reactivación económica al tiempo que se garantiza la protección del medio ambiente. En esta senda, el RD-ley 23/2020 había introducido una modificación de los artículos 34, 43 y 47 de la LEA, con objeto de regular la prórroga de vigencia de las declaraciones de impacto, evitando así el vacío jurídico existente y para agilizar el procedimiento para la determinación de alcance del estudio de impacto ambiental y el relativo a la evaluación 
ambiental simplificada, equiparándola al procedimiento de evaluación ambiental ordinaria ${ }^{6}$.

Ahora, el art. 66 del RD-ley 36/2020 interviene sobre el art. 8.3 de la LEA, artículo que regula la posibilidad, mediante acuerdo motivado del Consejo de Ministros o del órgano autonómico competente, en supuestos excepcionales, de excluir un proyecto determinado de la evaluación de impacto ambiental, cuando su aplicación pueda tener efectos perjudiciales para la finalidad del proyecto 0 aquellos proyectos que consistan en obras de reparación o mejora de infraestructuras críticas, definidas en la Ley 8/2011, de 28 de abril, por la que se establecen medidas para la protección de las infraestructuras críticas, que hayan sido dañadas como consecuencia de acontecimientos catastróficos o cuyo refuerzo sea necesario para garantizar la seguridad nacional.

Pues bien, el art. 66 RD-Ley 26/2020 introduce la previsión de considerar que concurren circunstancias excepcionales, para la aplicación del art. 8.3 LEA, en el caso de determinados proyectos financiados total o parcialmente mediante el Instrumento Europeo de Recuperación y, concretamente, cuando se trate de meras modernizaciones o mejoras de instalaciones ya existentes, que no supongan construcción de nueva planta, aumento de la superficie afectada 0 adición de nuevas construcciones ni afección sobre recursos hídricos y entre cuyos requisitos se incorporen para su financiación y aprobación la mejora de las condiciones ambientales, tales como la eficiencia energética o del empleo de recursos naturales, la reducción de su impacto ambiental o la mejora de la sostenibilidad de la instalación ya existente.

Por otro lado, la DF 3르 RD-ley 26/2020 reduce los plazos de resolución en las distintas fases del procedimiento de evaluación ambiental. En concreto, se reducen los plazos en el procedimiento de evaluación ambiental estratégica de planes y programas y de proyectos, estableciéndose adicionalmente una reducción de plazos en los procedimientos de evaluación de impacto ambiental simplificada y en los plazos para publicar o, en su caso, notificar, las resoluciones de los procedimientos de esta naturaleza.

\footnotetext{
${ }^{6}$ Se remite, en detalle, a la crónica anterior, "Legislación básica de protección del medio ambiente (segundo semestre 2020), Revista Catalana de Dret Ambiental, Vol. XI, Núm. 2 (2020), pp. 17 ss.
} 
Por otro lado, las Disposiciones Finales del RD-Ley modifican diversos artículos de normas ambientales y concretamente:

1. La DF 6⿳a modifica el art. 78 de la Ley $42 / 2007$, de 13 de diciembre, del Patrimonio Natural y de la Biodiversidad, para crear el Fondo de restauración ecológica y resiliencia (FRER) (FCPJ), con objeto de poner en práctica aquellas medidas destinadas a apoyar la consecución de los objetivos para lograr la transición a un modelo productivo y social más ecológico del Plan de recuperación, transformación y resiliencia en el ámbito de competencias del Ministerio para la Transición Ecológica y el Reto Demográfico, en materia de aguas y dominio público hidráulico; costas, protección y conservación del mar y del dominio público marítimoterrestre; cambio climático, su mitigación y adaptación y el fortalecimiento de la resiliencia climática; prevención de la contaminación, fomento del uso de tecnologías limpias y hábitos de consumo menos contaminantes y más sostenibles, de acuerdo con la política de economía circular; protección del patrimonio natural, de la biodiversidad y de los bosques; meteorología y climatología.

2. La DF $7^{a}$ modifica el art. 19.2 del Texto refundido de la Ley de prevención y control integrados de la contaminación, aprobado por Real Decreto Legislativo 1/2016, de 16 de diciembre. Concretamente el plazo establecido para el informe del Organismo de cuenca, en el ámbito del procedimiento para la concesión de autorización ambiental integrada, pasa a ser de 4 meses. Asimismo, modifica el art. 21 del Texto refundido, reduciendo de 9 a 6 meses el plazo para dictar la resolución, que tiene el órgano competente para otorgar la autorización ambiental integrada.

3. La DF $8^{\text {a }}$ modifica el Real Decreto 815/2013, de 18 de octubre, por el que se aprueba el Reglamento de emisiones industriales y de desarrollo de la Ley 16/2002, de 1 de julio, de prevención y control integrados de la contaminación. Concretamente se reducen los plazos previstos en el artículo 15, apartados 5 y 9 , relativos al procedimiento simplificado de modificación sustancial de autorización en la autorización ambiental integrada. 
4. La DF $10^{\mathrm{a}}$ modifica el art. 91 de la Ley $2 / 2011$, de 4 de marzo, de Economía Sostenible para crear el fondo «Fondo de carbono para una economía sostenible» (FES-CO2) (FCPJ), carente de personalidad jurídica, adscrito a la Secretaría de Estado de Medio Ambiente, que se dedicará a) al desarrollo de actuaciones adicionales de adaptación a los efectos del cambio climático con impacto significativo en la lucha contra el cambio climático; b) al desarrollo de actuaciones adicionales de reducción de emisiones de gases de efecto invernadero y aumento de sumideros de carbono, basándose en el precio de tonelada de $\mathrm{CO} 2$ equivalente reducida o absorbida; a) al apoyo a proyectos emblemáticos de desarrollo tecnológico con un potencial significativo para la descarbonización del sector de generación eléctrica o de la industria; d) a la adquisición de créditos de carbono, en especial los derivados de actividades realizadas o promovidas por empresas en el marco de los instrumentos de la Convención Marco de Naciones Unidas sobre el Cambio Climático y de su Acuerdo de París en los términos establecidos reglamentariamente, con la finalidad de incentivar la participación de las empresas españolas en dichos instrumentos. El Fondo se destinará de manera preferente a proyectos de eficiencia energética, energías renovables y gestión de residuos y a aquellos que representen un elevado componente de transferencia de tecnología en el país donde se lleven a cabo. Para la certificación de las reducciones de emisiones de las actividades se atenderá a las normas internacionales que las regulen, en función de su naturaleza.

Finalmente, rubricamos otras medidas que pueden ser de interés para nuestro ámbito:

1. Se introduce una nueva figura de colaboración público-privada: los Proyectos Estratégicos para la Recuperación y Transformación Económica (PERTES). El Capítulo III del Título II regula esta nueva fórmula más flexible de colaboración publico-privada, que tiene vocación de permanencia.

2. Se crean una serie de órganos y foros que prevén la participación de la sociedad civil, con el objetivo de impulsar los procesos participativos, así 
como órganos y comisiones de "gobernanza", en los que se prevé la participación de todos los Ministerios o de todas las administraciones: la Comisión para la Recuperación, Transformación y Resiliencia y la Conferencia Sectorial del Plan de Recuperación, Transformación y Resiliencia.

3. Desde un punto de vista procedimental (Capítulo II del Título IV), se declaran de tramitación urgente los procedimientos administrativos que estén vinculados a la ejecución de los fondos incluidos en el ámbito de aplicación de esta norma, por parte de la AGE. Por otro lado, se prevé una reducción de plazos, en el caso del procedimiento de suscripción de convenios por parte de la AGE y sus organismos públicos vinculados o dependientes, en cuanto a los informes que prevé el artículo 50.2 de la Ley 40/2015, de 1 de octubre, de Régimen Jurídico del Sector Público.

4. Se establecen especialidades en materia de contratación pública y de gestión de subvenciones, simplificando, en ambos casos, la tramitación de aquellas relacionadas con el uso de dichos fondos (Capítulo III y V del Título IV).

El RD-ley 26/2020 se vincula profundamente con algunas disposiciones de la Ley 11/2020, de 30 de diciembre, de Presupuestos Generales del Estado para el año $2021^{7}$. Debido a la imposibilidad de analizar en profundidad dicha norma, mencionamos solamente que la DA $132^{\mathrm{a}}$ prevé los ingresos procedentes de las subastas de derechos de emisión de gases de efecto invernadero con vigencia indefinida o hasta que entre en vigor la nueva Ley de Cambio Climático y Transición Energética. Por otro lado, la DA 152ª , autoriza al gobierno, para la elaboración de los planes, programas, estrategias, instrumentos y disposiciones de carácter general que se adopten en la lucha contra al cambio climático, a establecer Asambleas Ciudadanas del Cambio Climático en los tres niveles administrativos: Asamblea Nacional, Asambleas Autonómicas y Asambleas Municipales. Para su desarrollo e implementación se establece el plazo de un año. Cabe señalar también que la DA $115^{\text {a }}$ establece subvenciones a determinados municipios, por el servicio de transporte colectivo urbano, condicionadas por la aprobación de un Plan de Movilidad Sostenible.

\footnotetext{
${ }^{7}$ BOE núm. 341, de 31 de diciembre de 2020.
} 
En cuanto a las modificaciones, las Disposiciones finales modifican, entre otros, algunos artículos del

- Real Decreto 849/1986, de 11 de abril, por el que se aprueba el Reglamento del Dominio Público Hidráulico, que desarrolla los títulos preliminar, I, IV, V, VI y VII de la Ley 29/1985, de 2 de agosto, de Aguas.

- Texto refundido de la Ley de Aguas, aprobado por Real Decretolegislativo 1/2001, de 20 de julio (en concreto las funciones del presidente del Organismo de cuenca y el canon de regulación y tarifa de utilización del agua).

- Ley de Puertos del Estado y de la Marina Mercante, aprobado por Real Decreto Legislativo 2/2011, de 5 de septiembre (en concreto se prevé que, con el objeto impulsar actividades directamente conectadas a la descarbonización de la economía y a la adaptación al cambio climático, podrán aplicarse por la Autoridad Portuaria bonificaciones singulares, de hasta el 30 por ciento, cuando el objeto concesional sea la fabricación industrial de elementos destinados a instalaciones eólicas marinas).

\section{NOVEDADES NORMATIVAS EN ÁMBITOS SECTORIALES}

\subsection{En materia de energía eléctrica}

a) Estatuto de los consumidores electrointensivos

En cuanto a la normativa estatal aprobada, en el periodo de tiempo que abarca esta crónica, en el ámbito de energía eléctrica, destacamos el Real Decreto 1106/2020, de 15 de diciembre, por el que se regula el Estatuto de los consumidores electrointensivos ${ }^{8}$.

El art. 4 del Real Decreto-ley 20/2018, de 7 de diciembre, de medidas urgentes para el impulso de la competitividad económica en el sector de la industria y el comercio en España, contenía un mandato al Gobierno para que, en un plazo de seis meses, mediante real decreto, elaborara y aprobara un Estatuto de los Consumidores Electrointensivos, con el objetivo de reconocer las

${ }^{8}$ BOE núm. 328, de 17 de diciembre de 2020. 
particularidades y regular el régimen de aquellos consumidores eléctricos con un elevado uso de la electricidad, un elevado consumo en horas de baja demanda eléctrica y una curva de consumo estable y predecible.

La norma objeto de análisis regula los requisitos para poder optar a la categoría de consumidor electrointensivo, el procedimiento para obtener la certificación de la condición, así como las obligaciones de dichos consumidores (arts. 10-14). Finalmente, también se regulan las ayudas y el mecanismo de cobertura de riesgos derivados de la adquisición a medio y largo plazo de energía eléctrica por consumidores electrointensivos (arts. 38-48). El Anexo contiene el listado de sectores y actividades que pueden optar por esta condición. El Real Decreto 1106/2020 desarrolla lo dispuesto en el título III del Real Decreto-ley 24/2020, de 26 de junio, de medidas sociales de reactivación del empleo y protección del trabajo autónomo y de competitividad del sector industrial y modifica el Reglamento de Ordenación y Supervisión de Seguros Privados, aprobado por el Real Decreto 2486/1998, de 20 de noviembre.

b) Acceso y conexión a las redes de transporte y distribución de energía eléctrica

Otra norma de fundamental importancia relativa al sector de la energía eléctrica resulta ser el Real Decreto 1183/2020, de 29 de diciembre, de acceso y conexión a las redes de transporte y distribución de energía eléctrica ${ }^{9}$. Debe traerse a colación que el art. 33 de la Ley $24 / 2013$, de 26 de diciembre, del Sector Eléctrico, (LSE) regula los derechos y los permisos de acceso y conexión a la red eléctrica. Sin embargo, la aplicación de dicho artículo estaba supeditado al desarrollo reglamentario de los criterios para la concesión de los permisos de acceso y conexión (DT 11 $1^{\text {a }}$ LSE). Asimismo, la DT 1를 del Decreto-Ley 23/2020, de 23 de junio, por el que se aprueban medidas en materia de energía y en otros ámbitos para la reactivación económica, había introducido una moratoria a las nuevas solicitudes de permisos de acceso y conexión para plantas de producción de energía eléctrica, hasta la aprobación del Real Decreto objeto de análisis. Pues a través del Real Decreto 1183/2020, de 29 de diciembre, de acceso y

${ }^{9}$ BOE núm. 340, de 30 de diciembre de 2020. 
conexión a las redes de transporte y distribución de energía eléctrica se desarrolla el art. 33 LSE y se establecen los principios, criterios y el procedimiento de los permisos de acceso y de conexión a las redes de transporte y distribución de energía eléctrica que se aplicarán a productores, consumidores, titulares de instalaciones de almacenamiento y titulares y gestores de las redes de transporte y distribución. En cambio, están exentos de la obligación de solicitar y obtener los permisos de acceso y de conexión los titulares de las instalaciones de autoconsumo acogidos a la modalidad sin excedentes y a las con excedentes de potencia igual o inferior a $15 \mathrm{~kW}$, que se ubiquen en suelo urbanizado que cuente con las dotaciones y servicios requeridos por la legislación urbanística (DA 2 ${ }^{\mathrm{a}}$ Real Decreto-ley 15/2018, de 5 de octubre, de medidas urgentes para la transición energética y la protección de los consumidores). Asimismo, están exentos los consumidores que cumplan los requisitos establecidos en el artículo 25.1 del Real Decreto 1048/2013, de 27 de diciembre. El Real Decreto 1183/2020 modifica y deroga parcialmente el Real Decreto 1955/2000, de 1 de diciembre, por el que se regulan las actividades de transporte, distribución, comercialización, suministro y procedimientos de autorización de instalaciones de energía eléctrica y deroga parcialmente el Real Decreto 1699/2011, de 18 de noviembre, por el que se regula la conexión a red de instalaciones de producción de energía eléctrica de pequeña potencia. Por otro lado, como se ha adelantado, la aprobación del Real Decreto permite la aplicación del art. 33 LSE.

Al mismo cometido responde la Circular 1/2021, de 20 de enero, de la Comisión Nacional de los Mercados y la Competencia, por la que se establece la metodología y condiciones del acceso y de la conexión a las redes de transporte y distribución de las instalaciones de producción de energía eléctrica ${ }^{10}$. Recordamos que el apartado 11 del artículo 33 LSE, tras su modificación por el Real Decreto-ley 1/2019, de 11 de enero"11, establece que "la Comisión Nacional de los Mercados y la Competencia aprobará mediante Circular la metodología y

\footnotetext{
${ }^{10}$ BOE núm. 19, de 22 de enero de 2021.

${ }^{11}$ De medidas urgentes para adecuar las competencias de la Comisión Nacional de los Mercados y la Competencia a las exigencias derivadas del derecho comunitario en relación a las Directivas 2009/72/CE y 2009/73/CE del Parlamento Europeo y del Consejo, de 13 de julio de 2009, sobre normas comunes para el mercado interior de la electricidad y del gas natural.
} 
las condiciones de acceso y conexión que comprenderá: el contenido de las solicitudes y permisos, los criterios económicos, los criterios para la evaluación de la capacidad, los motivos para la denegación, el contenido mínimo de los contratos y la obligación de publicidad y transparencia de la información relevante para el acceso y la conexión". Así pues, la Circular 1/2021 tiene por objeto culminar el proceso de regulación del acceso y conexión a las redes de transporte y distribución de energía eléctrica, concretamente estableciendo la metodología y las condiciones de acceso y conexión a las redes de transporte y distribución por parte de los productores de energía eléctrica, incluidas las instalaciones de almacenamiento en los términos previstos en el artículo $6.3 \mathrm{del}$ Real Decreto 1183/2020, de 29 de diciembre. Con la aprobación de la Circular, resultan inaplicables las disposiciones anteriores al Real Decreto-ley 1/2019, de 11 de enero, que regulaban la metodología y condiciones de acceso y conexión a las redes de transporte y distribución de las instalaciones de producción de electricidad.

\section{c) Metodología de calculo de los cargos del sistema eléctrico}

Siempre en materia de energía eléctrica, hay que hacer una somera mención a la nueva normativa que regula la metodología de cálculo de los cargos del sistema eléctrico. En esta senda, recordamos que Real Decreto-ley 1/2019, de 11 de enero, modificó la LSE, estableciendo, por un lado, la competencia de la Administración General del Estado para regular la estructura de los cargos por costes regulados y de los cargos necesarios para cubrir otros costes del sistema. Y por el otro que el Gobierno, previo informe de la Comisión Nacional de los Mercados y la Competencia, tendrá que establecer la estructura y la metodología de cálculo de los cargos, mientras que la persona titular del Ministerio para la Transición Ecológica y el Reto Demográfico, previo acuerdo de la Comisión Delegada del Gobierno para Asuntos Económicos, tendrá que aprobar los cargos. Así pues, el Real Decreto 148/2021, de 9 de marzo, por el que se establece la metodología de cálculo de los cargos del sistema eléctrico ${ }^{12}$, tiene por objeto definir la metodología a emplear en el cálculo anual del reparto entre

${ }^{12}$ BOE núm. 66, de 18 de marzo de 2021. 
los usuarios del sistema eléctrico de la cuantía de los cargos del sistema (tal y como se ha determinado por la DF $3^{\underline{a}}$ del Real Decreto-ley 1/2019). Téngase en cuenta que la norma no establece los cargos, sino la metodología para calcularlos. En la elaboración de la metodología, en concreto, se han aplicado criterios de reparto que distorsionen lo menos posible la demanda global, que sean objetivos y no discriminatorios, aplicando los principios de transparencia en el cálculo y de simplicidad en su aplicación. Además, la metodología está alineada con los objetivos del Gobierno de descarbonización de la economía, transición justa, y fomento de la competitividad de la industria, e intenta contribuir a mantener la sostenibilidad económica y financiera del sistema eléctrico.

Por otro lado, siempre en referencia con la parte regulada de la factura eléctrica, cabe traer a colación la Circular 3/2021, de 17 de marzo, de la Comisión Nacional de los Mercados y la Competencia, por la que se modifica la Circular 3/2020, de 15 de enero, por la que se establece la metodología para el cálculo de los peajes de transporte y distribución de electricidad.

\section{d) Otras disposiciones en materia de energía eléctrica}

Además de las normas analizadas, siempre en materia de energía eléctrica, mencionamos las siguientes disposiciones:

- Orden TED/1271/2020, de 22 de diciembre, por la que se establecen diversos costes regulados del sistema eléctrico para el ejercicio 2021 y se prorrogan los peajes de acceso de energía eléctrica a partir del 1 de enero de $2021^{13}$.

- Orden TED/1161/2020, de 4 de diciembre, por la que se regula el primer mecanismo de subasta para el otorgamiento del régimen económico de energías renovables y se establece el calendario indicativo para el periodo 2020-2025 $5^{14}$.

- Orden TED/203/2021, de 26 de febrero, por la que se ejecutan diversas sentencias del Tribunal Supremo en relación con la retribución de las empresas de distribución de energía eléctrica para el año $2016^{15}$.

\footnotetext{
${ }^{13}$ BOE núm. 339, de 29 de diciembre de 2020.

${ }^{14}$ BOE núm. 318, de 5 de diciembre de 2020.

${ }^{15}$ BOE núm. 57, de 8 de marzo de 2021.
} 
- Orden TED/257/2021, de 18 de marzo, por la que se establecen los valores de la retribución a la operación correspondientes al segundo semestre natural del año 2019, aplicables a determinadas instalaciones de producción de energía eléctrica a partir de fuentes de energía renovables, cogeneración y residuos ${ }^{16}$.

- Orden TED/260/2021, de 18 de marzo, por la que se adoptan medidas de acompañamiento a las instalaciones cuyos costes de explotación dependen esencialmente del precio del combustible durante el periodo de vigencia del estado de alarma debido a la situación de crisis sanitaria ocasionada por el COVID-1917.

- Circular 2/2021, de 10 de febrero, de la Comisión Nacional de los Mercados y la Competencia, por la que se establece la metodología y condiciones del etiquetado de la electricidad para informar sobre el origen de la electricidad consumida y su impacto sobre el medio ambiente ${ }^{18}$.

\subsection{En materia de contaminación atmosférica}

En materia de contaminación atmosférica, en el semestre de referencia, deben resaltarse dos normas importantes.

a) Real Decreto 1089/2020, de 9 de diciembre, por el que se desarrollan aspectos relativos al ajuste de la asignación gratuita de derechos de emisión de gases de efecto invernadero en el periodo 2021-203019.

En 2018, la Unión Europea adoptó la Directiva (UE) 2018/410 del Parlamento Europeo y del Consejo, de 14 de marzo de 2018, por la que se modifica la Directiva 2003/87/CE para intensificar las reducciones de emisiones de forma eficaz en relación con los costes y facilitar las inversiones en tecnologías hipocarbónicas, así como la Decisión (UE) 2015/1814. La Directiva constituye el marco legislativo de la Unión Europea para el periodo de comercio 2021-2030 (fase IV) del RCDE UE y se configura como uno de los instrumentos principales

\footnotetext{
${ }^{16}$ BOE núm. 68, de 18 de marzo de 2021.

${ }^{17}$ BOE núm. 69, de 22 de marzo de 2021.

${ }^{18}$ BOE núm. 43, de 19 de febrero de 2021.

${ }^{19}$ BOE núm. 322, de 10 de diciembre de 2020.
} 
de la Unión Europea para alcanzar sus objetivos de reducción de emisiones en 2030. Dicha Directiva tiene como objetivo intensificar la reducción de emisiones en el cuarto periodo de comercio del RCDE UE, que abarca los años 2021-2030 $y$, por ello, mejorar la coherencia entre la asignación gratuita de derechos de emisión para las instalaciones fijas y sus niveles de actividad reales, intentando reducir, además, en la medida de lo posible, el exceso de las cargas administrativas a los titulares de las instalaciones. Así pues, la Directiva establece la necesidad de ajustarse de forma simétrica para las asignaciones de derechos de emisión de las instalaciones cuyas operaciones -de acuerdo con evaluaciones sobre la base de un promedio móvil de dos años (nivel medio de actividad) - hayan aumentado o disminuido más del quince por ciento en comparación con el nivel utilizado inicialmente para determinar la asignación gratuita de derechos de emisión en el periodo de asignación pertinente (nivel histórico de actividad). Las reglas específicas para el ajuste de la asignación gratuita han sido concretadas y desarrolladas en el Reglamento de Ejecución (UE) 2019/1842 de la Comisión, de 31 de octubre de 2019, por el que se establecen disposiciones de aplicación de la Directiva 2003/87/CE del Parlamento Europeo y del Consejo respecto de las disposiciones adicionales de ajuste de la asignación gratuita de derechos de emisión debido a modificaciones del nivel de actividad.

En esta senda, el objeto del Real Decreto 1089/2020 es la determinación de los aspectos relacionados con el ajuste anual de la asignación gratuita de derechos de emisión de las instalaciones fijas durante el periodo de comercio 2021-2030, incluidas las obligaciones de información (Capítulo II), y otros aspectos derivados del ajuste, como son la devolución de derechos. El Real Decreto transpone los apartados 17 y 14. m) y n), respectivamente, del artículo 1 de la Directiva (UE) 2018/410 del Parlamento Europeo y del Consejo, de 14 de marzo de 2018, que introducen las circunstancias determinantes del ajuste de la asignación y las obligaciones de información.

b) Ley $9 / 2020$, de 16 de diciembre, por la que se modifica la Ley $1 / 2005$, de 9 de marzo, por la que se regula el régimen del comercio de derechos de 
emisión de gases de efecto invernadero, para intensificar las reducciones de emisiones de forma eficaz en relación con los $\operatorname{costes}^{20}$.

Siempre en el ámbito de las novedades relativas al RDCE UE, para el nuevo periodo de comercio señalado, 2021-2030, se ha aprobado la Ley 9/2020, de 16 de diciembre, que modifica la Ley $1 / 2005$, de 9 de marzo, por la que se regula el régimen del comercio de derechos de emisión de gases de efecto invernadero, para intensificar las reducciones de emisiones de forma eficaz en relación con los costes. Dicha modificación tiene como objetivo la incorporación parcial al ordenamiento jurídico español de la Directiva (UE) 2018/410 del Parlamento Europeo y del Consejo, de 14 de marzo de 2018.

Entre las novedades introducidas por la Ley 9/2020, en la Ley $1 / 2005$, señalamos,

- en lo relativo a las autorizaciones de emisión de gases de efecto invernadero con las que deben contar las instalaciones sometidas al ámbito de aplicación de la Ley (Cap. II), la eliminación de la obligación por parte del órgano autonómico de revisar cada cinco años la autorización de emisión de gases de efecto invernadero.

- En lo relativo a la regulación de los derechos de emisiones (Cap. III), los derechos de emisión expedidos a partir del 1 de enero de 2013 tendrán una validez indefinida y tienen la consideración de instrumentos financieros conforme a la normativa nacional y de la Unión Europea que resulte de aplicación. Por otro lado, la duración de los periodos de comercio de derechos de emisión podrá comprender más de un periodo de asignación, en relación con la asignación gratuita. En esta senda, la fase IV del RCDE UE se divide, para las instalaciones fijas, en dos periodos de asignación que abarcan respectivamente los años 2021-2025 y 2026-2030.

- En cuanto a la asignación de derechos de emisión (Cap. IV), el titular de la Secretaría de Estado de Medio Ambiente, que es el órgano encargado de la organización de las subastas de derechos de emisión de gases de efecto invernadero, ejercerá la función de subastador.

${ }^{20}$ BOE núm. 328, de 17 de diciembre de 2020. 
- Se introduce un nuevo capítulo (el V) dedicado a la regulación de los ajustes y de la devolución de la asignación gratuita de derechos de emisión. En concreto, se establece que la cantidad asignada gratuitamente a cada instalación deberá ser ajustada de acuerdo con el nivel de actividad de la instalación determinado sobre la base de un promedio móvil de dos años, cuando la variación de dicho nivel de actividad, sea al alza o a la baja, supere el quince por ciento en comparación con el nivel de actividad utilizado inicialmente para determinar la asignación gratuita en el periodo de asignación.

- En cuanto a las obligaciones de seguimiento y notificación de las emisiones para las instalaciones fijas y de los niveles de actividad (Cap. VI), se establece la nueva obligación, para las instalaciones que reciben asignación gratuita de derechos de emisión, de llevar a cabo un seguimiento de los niveles de actividad de las subinstalaciones en las que esté dividida cada instalación.

- Respecto a las disposiciones adicionales de la Ley 1/2005, de 9 de marzo, destacamos la modificación efectuada en la DA $4^{a}$, por la que se regula la exclusión de instalaciones de pequeño tamaño, durante el periodo de asignación 2021 a 2025. No obstante, se redefine el contenido mínimo de la medida equivalente con base en los nuevos objetivos de reducción de emisiones, a cuyo efecto se ha aprobado el Real Decreto 317/2019, de 26 de abril de 2019.

\subsection{En materia de residuos}

En materia de residuos, destacamos la aprobación del Real Decreto 27/2021, de 19 de enero, por el que se modifican el Real Decreto 106/2008, de 1 de febrero, sobre pilas y acumuladores y la gestión ambiental de sus residuos, y el Real Decreto 110/2015, de 20 de febrero, sobre residuos de aparatos eléctricos y electrónicos ${ }^{21}$. El Real Decreto objeto de análisis tiene como objetivo trasponer la Directiva (UE) 2018/849 del Parlamento Europeo y del Consejo, de 30 de mayo

${ }^{21}$ BOE núm. 17, de 20 de enero de 2021. 
de $2018^{22}$ y pretende mejorar la gestión de los residuos de pilas y acumuladores y de los RAEE estableciendo normas más claras que incrementen el nivel de seguridad jurídica. En este sentido, el artículo primero modifica el Real Decreto 106/2008, de 1 de febrero, para introducir los nuevos códigos LER (Lista Europea de Residuos) para la identificación de residuos de pilas, acumuladores y baterías considerados peligrosos. Asimismo, introduce la necesidad de incentivar el principio de jerarquía de residuos (art. 5 bis). El artículo segundo, en cambio, modifica en profundidad el Real Decreto 110/2015, de 20 de febrero. En sus cuarenta y tres apartados, se recogen, por un lado, las disposiciones que permiten transponer la mencionada Directiva 2018/849 y, por el otro, una serie de disposiciones que introducen mejoras en la citada norma. Entre todas, cabe mencionar la modificación del apartado cuarto del artículo 7 del Real Decreto 110/2015, para especificar que los costes de recogida, tratamiento y eliminación de los RAEE (residuos de aparatos eléctricos y electrónicos) en los que anualmente hubieran incurrido los productores de AEE (aparatos eléctricos y electrónicos) no figuren en las facturas que impliquen la comercialización de un $A E E$, en cualquier punto de la cadena. Asimismo, hay que destacar la modificación del artículo 38 con el objetivo de clarificar el papel de los productores de AEE en el ámbito de su responsabilidad, especificando de un modo más detallado las responsabilidades que asumen. Finalmente, destacamos la extensión del ámbito de aplicación del Real Decreto a todos los $A E E$, que se clasifican en las categorías recogidas en su anexo III. El Real Decreto en objeto, además de las dos normas mencionadas, modifica puntualmente el Real Decreto 646/2020, de 7 de julio, por el que se regula la eliminación de residuos mediante depósito en vertedero (DF $1^{1}$ ) y el Real Decreto 818/2018, de 6 de julio, sobre medidas para la reducción de las emisiones nacionales de determinados contaminantes atmosféricos (DF $2^{\mathfrak{a}}$ ).

\footnotetext{
${ }^{22}$ La DF $2^{\text {a }}$ incorpora también parte de la Directiva 2016/2284 del Parlamento Europeo y del Consejo, de 14 de diciembre de 2016, relativa a la reducción de las emisiones nacionales de determinados contaminantes atmosféricos, por la que se modifica la Directiva 2003/35/CE y se deroga la Directiva 2001/81/CE, mediante la modificación del Real Decreto 818/2018, de 6 de julio.
} 


\subsection{En materia de instalaciones térmicas en edificios}

En materia de instalaciones térmicas en edificios es preciso mencionar el Real Decreto 178/2021, de 23 de marzo, por el que se modifica el Real Decreto 1027/2007, de 20 de julio, por el que se aprueba el Reglamento de Instalaciones Térmicas en los Edificios ${ }^{23}$ (RITE). La norma objeto de análisis introduce una revisión del RITE para trasponer parcialmente la regulación de las instalaciones técnicas en los edificios prevista en la Directiva (UE) 2018/844 del Parlamento Europeo y del Consejo, de 30 de mayo de 2018, por la que se modifica la Directiva 2010/31/UE relativa a la eficiencia energética de los edificios y la Directiva 2012/27/UE relativa a la eficiencia energética; y la regulación de las instalaciones técnicas en los edificios prevista en la Directiva (UE) 2018/2002 del Parlamento Europeo y del Consejo, de 11 de diciembre de 2018, por la que se modifica la Directiva 2012/27/UE relativa a la eficiencia energética.

Entre otras, destacamos las siguientes novedades:

- La instalación en edificaciones de sistemas térmicos convencionales en lugar de otros más eficientes y sostenibles, necesitará de una justificación que deberá ir acompañada de una comparativa entre el sistema de producción de energía elegido con otros alternativos, teniendo en cuenta los sistemas que sean viables técnica, medioambiental y económicamente, en función del clima y de las características específicas del edificio y su entorno.

- Queda prohibida la utilización de combustibles sólidos de origen fósil en las instalaciones térmicas de los edificios de nueva construcción y en las instalaciones térmicas que se reformen en los edificios existentes.

- En cuanto a la incorporación de energía renovable en los edificios, se establece que, en todas las edificaciones sujetas a reforma, el técnico competente propondrá instalaciones alternativas de alta eficiencia, siempre que ello sea técnica, funcional y económicamente viable y siempre que se cumplan los requisitos de condiciones climáticas interiores saludables, la seguridad contra incendios y los riesgos relacionados con una intensa actividad sísmica.

${ }^{23}$ BOE núm. 71, de 24 de marzo de 2021. 
- Los edificios no residenciales con grandes consumos, es decir los edificios con una potencia útil nominal de climatización superior a $290 \mathrm{~kW}$, deberán estar equipados, a más tardar en 2025, con sistemas de automatización y control de edificios con el objetivo de monitorizar y racionalizar el consumo energético y reducir la emisión de gases de efecto invernadero a la atmósfera.

- Asimismo, la modificación del RITE introduce obligaciones acerca de los contadores de agua caliente sanitaria (ACS) para redes urbanas (T 1.2.4.1.2.4).

La modificación del RITE responde a la exigencia de alcanzar los objetivos climáticos establecidos en el Plan Nacional Integrado de Energía y Clima (PNIEC) 2021 y, en concreto, el referido a la mejora de la eficiencia energética a través de la reducción del consumo de energía primaria en un 39,5\% en 2030.

\section{ULTERIORES DISPOSICIONES DE INTERÉS PARA LA PROTECCIÓN DEL MEDIO AMBIENTE}

Por último, señalamos las siguientes disposiciones, que pueden resultar de interés para la protección del medio ambiente:

- Ley Orgánica 3/2020, de 29 de diciembre, por la que se modifica la Ley Orgánica 2/2006, de 3 de mayo, de Educación ${ }^{24}$. En la Ley se hace hincapié en la necesidad de que el sistema educativo tome en consideración los desafíos que plantea el cambio climático del planeta, por lo que los centros docentes han de convertirse en un lugar de custodia y cuidado de nuestro medio ambiente y han de promover una cultura de la sostenibilidad ambiental, de la cooperación social, desarrollando programas de estilos de vida sostenible y fomentando el reciclaje y el contacto con los espacios verdes. En tal sentido, se modifican varios artículos de la LOE para introducir la consideración de la sostenibilidad ambiental y del cambio climático en la enseñanza.

- Real Decreto 179/2021, de 23 de marzo, por el que se aprueba el Estatuto del Instituto para la Transición Justa, O.A. ${ }^{25}$

${ }^{24}$ BOE núm. 340, de 30 de diciembre de 2020.

${ }^{25}$ BOE núm. 72, de 25 de marzo de 2021. 
- Orden TED/275/2021, de 18 de marzo, por la que se establecen las obligaciones de aportación al Fondo Nacional de Eficiencia Energética en el año $2021^{26}$.

- Orden TED/1126/2020, de 20 de noviembre, por la que se modifica el Anexo del Real Decreto 139/2011, de 4 de febrero, para el desarrollo del Listado de Especies Silvestres en Régimen de Protección Especial y del Catálogo Español de Especies Amenazadas, y el Anexo del Real Decreto 630/2013, de 2 de agosto, por el que se regula el Catálogo Español de Especies Exóticas Invasoras ${ }^{27}$.

Finalmente, en cuanto a las ayudas y subvenciones señalamos:

- Orden ICT/971/2020, de 15 de octubre, por la que se desarrolla el programa de renovación del parque circulante español en 2020 (Plan Renove 2020) y se modifica el Anexo II del Real Decreto-ley 25/2020, de 3 de julio, de medidas urgentes para apoyar la reactivación económica y el empleo ${ }^{28}$.

- Real Decreto 905/2020, de 13 de octubre, por el que se regula la concesión directa de subvenciones en el ámbito de la transición ecológica financiadas con cargo a la cuota íntegra del Impuesto de Sociedades ${ }^{29}$.

- Real Decreto 1186/2020, de 29 de diciembre, por el que se modifica el Real Decreto 263/2019, de 12 de abril, por el que se regula el Programa de ayudas para actuaciones de eficiencia energética en PYME y gran empresa del sector industrial ${ }^{30}$.

- Real Decreto 149/2021, de 9 de marzo, por el que se regula el programa de ayudas para la realización de actuaciones de eficiencia energética en explotaciones agropecuarias y se acuerda la concesión directa de las ayudas de este programa a las comunidades autónomas ${ }^{31}$.

\footnotetext{
${ }^{26}$ BOE núm. 72, de 25 de marzo de 2021.

${ }^{27}$ BOE núm. 314, de 1 de diciembre de 2020.

${ }^{28}$ BOE núm. 275, de 17 de octubre de 2020.

${ }^{29}$ BOE núm. 728, de 21 de octubre de 2020.

${ }^{30}$ BOE núm. 340, de 30 de diciembre de 2020 .

${ }^{31}$ BOE núm. 59, de 10 de marzo de 2021.
} 